\title{
Multiwavelength Observation of a New Black Hole Candidate: EXS 1737.9-2952
}

\section{Citation}

Durouchoux, Ph., O. Vilhu, S. Corbel, P. Wallyn, K. S. Dwarakanath, J. Huovelin, K. R.

Anantharamaiah, et al. 1998. "Multiwavelength Observation of a New Black Hole Candidate: EXS 1737.9-2952." The Astrophysical Journal 507 (2): 781-93. https://doi.org/10.1086/306337.

\section{Permanent link}

http://nrs.harvard.edu/urn-3:HUL.InstRepos:41399830

\section{Terms of Use}

This article was downloaded from Harvard University's DASH repository, and is made available under the terms and conditions applicable to Other Posted Material, as set forth at http:// nrs.harvard.edu/urn-3:HUL.InstRepos:dash.current.terms-of-use\#LAA

\section{Share Your Story}

The Harvard community has made this article openly available.

Please share how this access benefits you. Submit a story.

Accessibility 
THE Astrophysical JouRnal, 507:781-793, 1998 November 10

(C) 1998. The American Astronomical Society. All rights reserved. Printed in U.S.A.

\title{
MULTIWAVELENGTH OBSERVATION OF A NEW BLACK HOLE CANDIDATE: EXS 1737.9-2952
}

\author{
P. Durouchoux, ${ }^{1}$ O. Vilhu, ${ }^{2}$ S. Corbel, ${ }^{1}$ P. Wallyn, ${ }^{3}$ K. S. Dwarakanath, ${ }^{4}$ J. Huovelin, ${ }^{2}$ K. R. Anantharamaiah, ${ }^{4}$ \\ J. Grindlay, ${ }^{5}$ C. Chapuis, ${ }^{1}$ Y. S. ParK, ${ }^{6}$ J. Bally, ${ }^{7}$ AND W. MahONEY ${ }^{3}$ \\ Received 1995 November 15 ; accepted 1997 October 7
}

\begin{abstract}
We report a multiwavelength analysis of an unusual high-energy transient: EXS 1737.9-2952. Due to the features this source exhibited in the hard X-ray domain similar to another source in the Galactic center region (1E1740.9 - 2952), and in order to study the molecular gas toward this X-ray source, we performed an observation of the EXS region in 1993 August, using the Swedish-ESO Submillimeter Telescope (SEST) located in La Silla (Chilean Andes). We observed a cloud, at the "forbidden" velocity of $135 \mathrm{~km} \mathrm{~s}^{-1}$, using ${ }^{12} \mathrm{CO}(1-0)$ transitions, giving a maximum column density of $8 \times 10^{21} \mathrm{~cm}^{-2}$. In 1994 we conducted other observations to search for higher density regions inside the cloud, using $\mathrm{HCO}+$ and CS lines, but they were unsuccessful: we concluded that the cloud could be associated with the X-ray source and its mean density is of the order of $\approx 10^{3} \mathrm{~cm}^{-3}$. In 1994 April, we performed a set of observations of the field containing EXS, at $20 \mathrm{~cm}$ and $6 \mathrm{~cm}$, using the VLA in its A configuration, and found four possible radio candidates for an association with the EXS X-ray source, one of them (source 3) being extended, exhibiting a head-tail morphology, and a having a thermal spectrum with a spectral index $\approx-0.7$. We reproduced our observation in 1994 November and December, using the $\mathrm{C}$ configuration at $6 \mathrm{~cm}$, in order to investigate on possible variability and extension of these sources and found a marginal indication in the $20 \mathrm{~cm}$ image that source 3 may have a weak second component displaced about $15^{\prime \prime}$. Nevertheless, this indication is too faint to associate this source definitely with EXS since no significant variation was detected. In addition, during the 1994 November-December observation, two more extended sources were detected but their association with EXS is unlikely.

We also analyzed the Einstein/IPC image of the $5 \sigma$ EXS error box which does not exhibit, at the time of the observation, any significant low-energy X-ray counterpart to EXS. A nearby pulsar PSR 1737-30 in the ROSAT catalog is outside this error box. Finally, IRAS maps of the EXS region do not show any IR contribution at the location of the radio sources. We conclude that (1) EXS 1737.9-2952 is a highenergy transient, (2) a persistent counterpart at other wavelengths is not demonstrated, and (3) EXS, when flaring, as well as other GC gamma-ray sources, could possibly contribute to the $511 \mathrm{keV}$ bulge emission.
\end{abstract}

Subject headings: black hole physics — Galaxy: center — gamma rays: theory — ISM: molecules

\section{INTRODUCTION}

The Galactic center (GC) has been observed in hard $\mathrm{X}$-rays and soft gamma rays many times over the past two decades, but we still do not have a comprehensive picture of the physical processes involved and the various emission mechanisms observed in this region.

It is fairly well established that the narrow GC $511 \mathrm{keV}$ annihilation line originates from a steady diffuse emission coming mainly from SN I's, and very recently it has been proposed (Purcell et al. 1996) that the distribution consists in a three-component model: a Galactic disk, a nuclear bulge, and an extended halo. This third component, containing about $80 \%$ of the diffuse $511 \mathrm{keV}$ flux, is required to explain observations by large field-of-view instruments, mainly SMM, GRIS, and Hexagone. Moreover, OSSE 511 $\mathrm{keV}$ measurements (Purcell et al. 1996) show a significant

\footnotetext{
${ }^{1}$ C. E. Saclay, DSM, DAPNIA, Service d'Astrophysique, 91191 Gifsur-Yvette Cedex, France; durouchoux@sapvxg.saclay.cea.fr.

2 Observatory, Box 14, FIN-00014 University of Helsinki, Finland.

3 Jet Propulsion Laboratory, 169-327, California Institute of Technology, 4800 Oak Grove Drive Pasadena, CA 91109-8099.

${ }_{4}^{4}$ Raman Research Institute, Sadashivanagar, Bangalore 560 080, India.

${ }^{5}$ Center for Astrophysics, Harvard University/MS-6, 60 Garden Street, Cambridge, MA 02138.

${ }^{6}$ Daeduk Radio Telescope, San 36-1, Whaam, Yusong, Daejon, 305348 , Korea.

${ }^{7}$ University of Colorado, APAS, Campus box 391, Boulder, CO 80309.
}

offset from $1=0^{\circ}$, with a centroid consistent with $1 \mathrm{E}$ 1740.7-2942 (hereafter 1E). Fainter sources in the GC region, emitting positrons which annihilate, as 1E, in nearby clouds (Bally \& Leventhal 1991; Mirabel et al. 1991) could exist and also contribute to the $511 \mathrm{keV}$ distribution (Wallyn et al. 1996). All these considerations could lead either to a number of faint sources, occasionally flaring, possibly emitting a narrow line at the annihilation energy, but most of the time undetectable considering the sensitivities of the present instruments. A number of such candidates have been observed in X-rays by Granat-SIGMA over the past 3 years (GRS 1758-258, GX 5-1, GRS 1741-2851, GX 1+4, A1742-294, Terzan 2, etc.). Moreover, some of them could be black holes, fed either by a companion or a nearby dense cloud. It has been shown (Shakura \& Sunyaev 1973) that instabilities in the accretion onto a compact object can increase the accretion rate, and the innermost part of the disk can reach relativistic temperatures, making pair plasma creation possible. An alternative mechanism to explain the creation of positrons is the upscattering of soft X-ray photons (created in the accretion disk) by hot electrons, leading to gamma-rays which interact via $\gamma-\gamma$ reactions and produce a pair plasma. A small fraction of these positrons can escape from the disk (Liang 1991) and annihilate in the interstellar medium (ISM). In the case of 1E, Zdziarski et al. (1994) have shown that the annihilation could take place in the jets. 


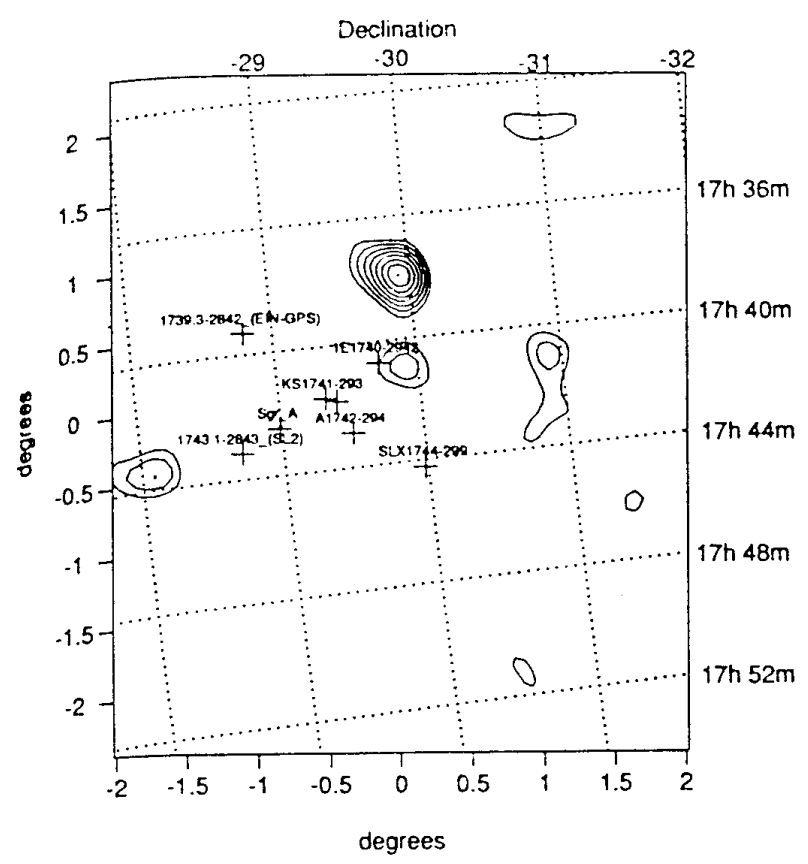

FIG. $1 a$

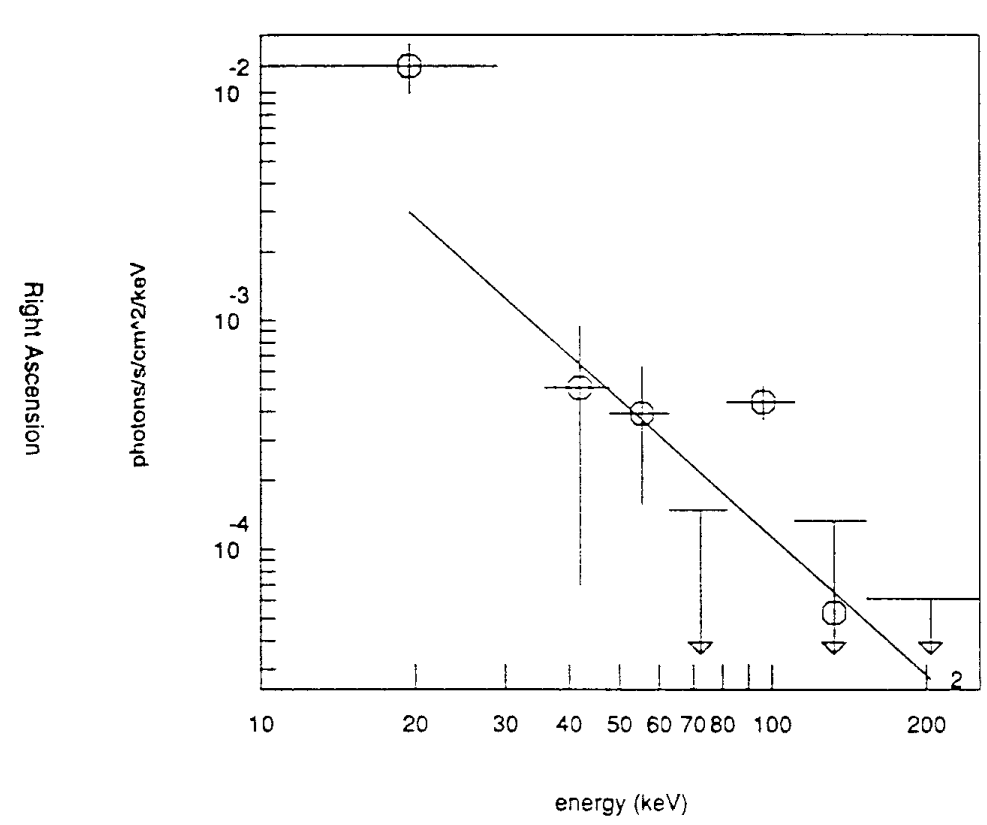

FIG. $1 b$

Fig. 1.-(a) Narrowband image (83-111 keV) of the Galactic center region showing $\geq 5.5 \sigma$ detection of EXS 1737.9-2952 (Grindlay et al. 1993). (b) Spectrum of the possible transient EXS $1739.9-2952$ showing detection at both $\approx 100 \mathrm{keV}$ and soft $(\approx 20-30 \mathrm{keV}) \mathrm{X}-\mathrm{rays}$.

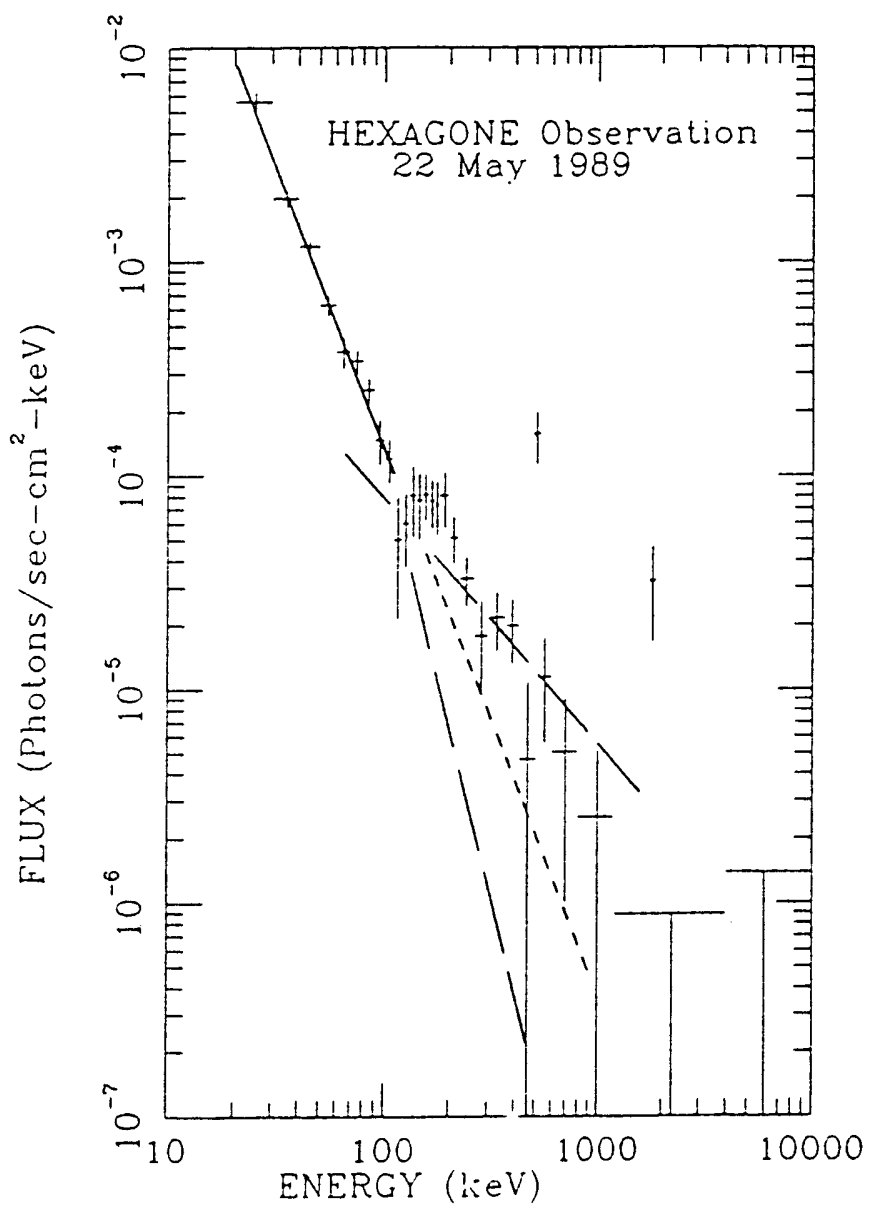

FIG. 2.- Spectrum of the Galactic center region including EXS, measured by HEXAGONE on 1989 May 22. Solid line indicates the power law that gives the best fit to the $20-120 \mathrm{keV}$ data. This is extended to higher energies as the short dashed line. The spectrum above $120 \mathrm{keV}$ clearly lies well above this.
In 1989 May, the Harvard Center for Astrophysics imaging balloon X-ray telescope (Exite) was flown from Alice Springs (Australia) and observed the GC region for about 2 hours. Two sources were detected: $1 \mathrm{E}$ and a new source EXS 1737.9-2952 (EXS hereafter), 40' from 1E, which exhibited an excess in the $83-111 \mathrm{keV}$ energy band (Figs. $1 a$ and $1 b$ ), interpreted as $102 \mathrm{keV}$ double-scattered annihilation photons (Grindlay, Covault, \& Manandhar 1993). A few days later, a US/French balloon experiment, Hexagone, with no imaging capabilities, but using highenergy resolution semiconductor detectors, measured a narrow annihilation line and a $170 \mathrm{keV}$ feature (Fig. 2) superimposed on the continuum spectrum in its $20^{\circ}$ FWHM field of view (Matteson et al. 1993), attributed to $511 \mathrm{keV}$ backscattered annihilation photons (Lingenfelter \& Hua 1991). Wallyn \& Durouchoux (1994) have pointed out a possible correlation between the 102 and $170 \mathrm{keV}$ lines, which could originate from the same source (EXS?).

We present in $\S 2$ a radio observation of the EXS region at $20 \mathrm{~cm}$ and $6 \mathrm{~cm}$ in order to search for a radio counterpart and possible radio structures, as seen in 1E and GRS $1758-258$ sources. In $\S 3$, we show the results of a search for a molecular cloud in the line of sight of EXS, using CO, ${ }^{13} \mathrm{CO}, \mathrm{HCO}^{+}$, and $\mathrm{CS}$ radio lines. In $\S 4$, we analyze Einstein and IRAS maps of the EXS region in order to search for a possible counterpart in low-energy X-rays and far-IR, respectively. Finally, we discuss the results and present a summary of this study in $\S 5$.

\section{VLA OBSERVATIONS OF EXS $1737.9-2952$}

The first set of observations of the field containing EXS 1737.9-2952 was made 1994 April 3 and 8, using the VLA in its A configuration, which has maximum baselines up to $35 \mathrm{~km}$. On both days, observations were made in the 20 and $6 \mathrm{~cm}$ bands. The pointing center for all the observations was $\alpha_{1950}=17^{\mathrm{h}} 37^{\mathrm{m}} 52^{\mathrm{s}}$ and $\delta_{1950}=-29^{\circ} 52^{\prime}$. The field of view 


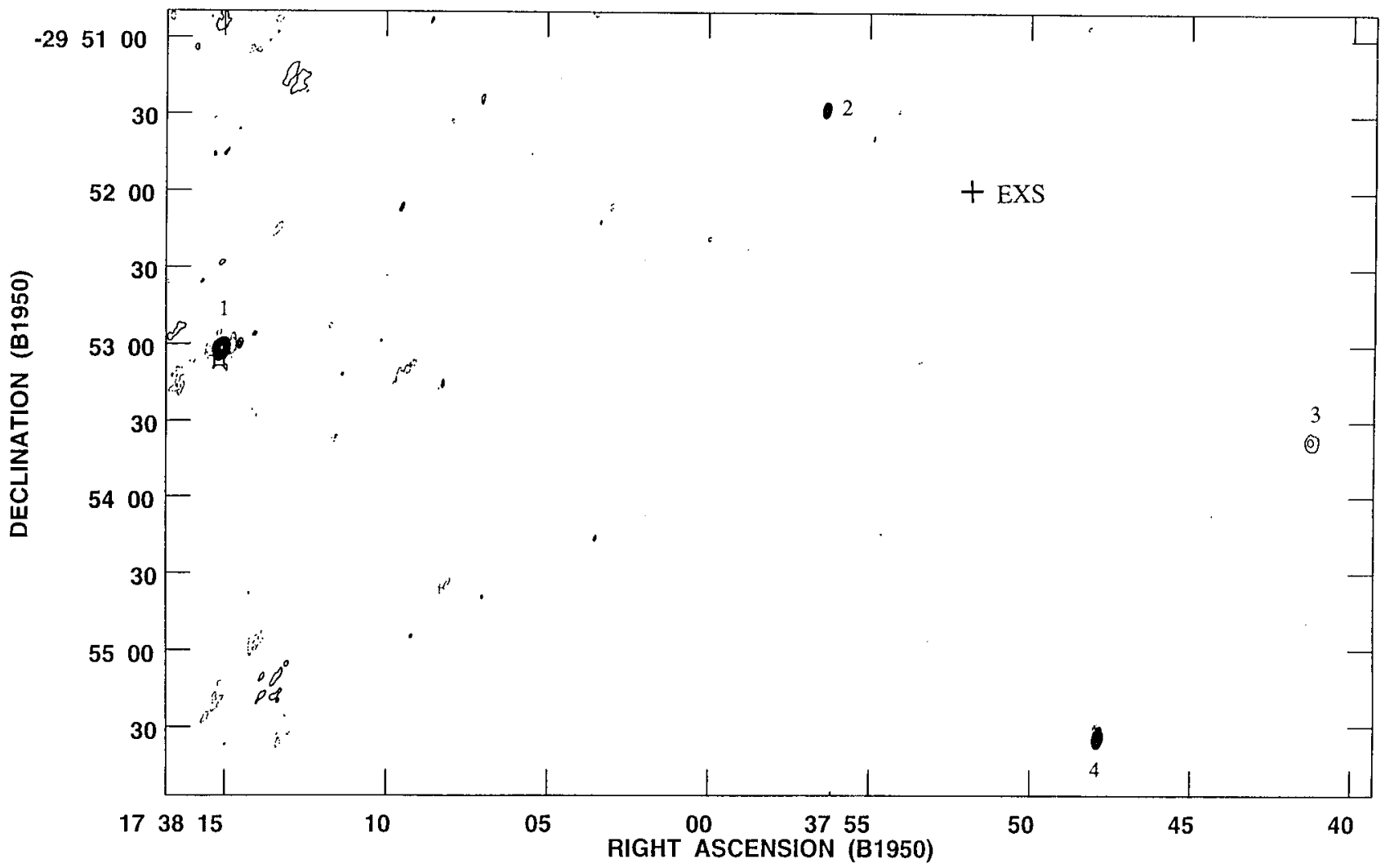

FIG. 3. - $20 \mathrm{~cm}$ image of the region containing X-ray source EXS 1737.9-2952 from 1994 April observations. The central position of the X-ray source is marked with a plus sign. The positional error radius of the X-ray source is $10^{\prime}$. The contours are $-0.6,-0.3,0.3,0.6,1.2,1.5,2.25,3,6,9$, and $12 \mathrm{mJy}$ beam ${ }^{-1}$. Sources 1,2,3, and 4 are clockwise starting from the strongest source located toward the east (source labeled 1 on the figure).

TABLE 1

VLA OBSERVATIONS

\begin{tabular}{|c|c|c|c|c|c|c|}
\hline $\begin{array}{l}\text { Image } \\
(\mathrm{cm})\end{array}$ & Date & $\begin{array}{l}\text { Duration } \\
\quad(\mathrm{hr})\end{array}$ & $\begin{array}{l}\text { Flux Density } \\
\left(\text { Jy beam }^{-1}\right)\end{array}$ & $\begin{array}{l}\text { Synthesized Beam } \\
(\operatorname{arcsec})\end{array}$ & $(\mathrm{deg})$ & $\begin{array}{c}\text { RMS } \\
\left(\mathrm{mJ} \mathrm{beam}^{-1}\right)\end{array}$ \\
\hline $20 \ldots \ldots$ & 1994 Apr & 4 & $\begin{array}{l}1.25(0.007) \\
1.20(0.007)\end{array}$ & $3.39 \times 1.59$ & 4.65 & 77 \\
\hline $6 \ldots \ldots$ & 1994 Apr & 4 & $\begin{array}{l}0.486(0.002) \\
0.489(0.002)\end{array}$ & $1.40 \times 1.10$ & 53.0 & 23 \\
\hline $6 \ldots \ldots$ & 1994 Nov & 3 & $\begin{array}{l}0.473(0.001) \\
0.476(0.001)\end{array}$ & $8.60 \times 5.05$ & 14.5 & 32 \\
\hline $6 \ldots \ldots$ & $1994 \mathrm{Dec}$ & 4 & $\begin{array}{l}0.466(0.001) \\
0.471(0.001)\end{array}$ & $8.25 \times 5.07$ & 16.0 & 29 \\
\hline
\end{tabular}

NoTE.-Numbers in parentheses are $1 \sigma$ formal errors. The two flux densities for each observation correspond to the two center frequencies within each band as mentioned in the text.

TABLE 2

Parameters of Sources in Figures 3 AND 5

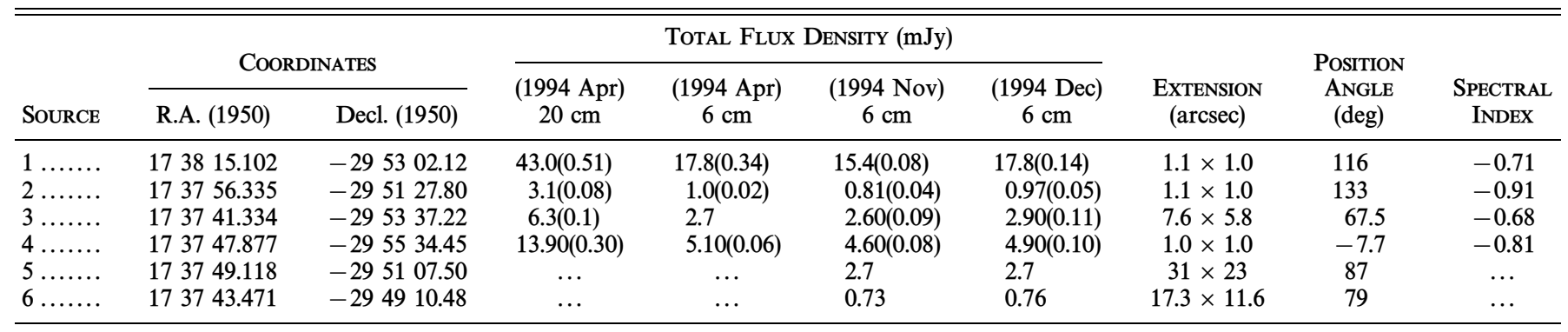

NotE.-Numbers in the parentheses are $1 \sigma$ formal errors. 


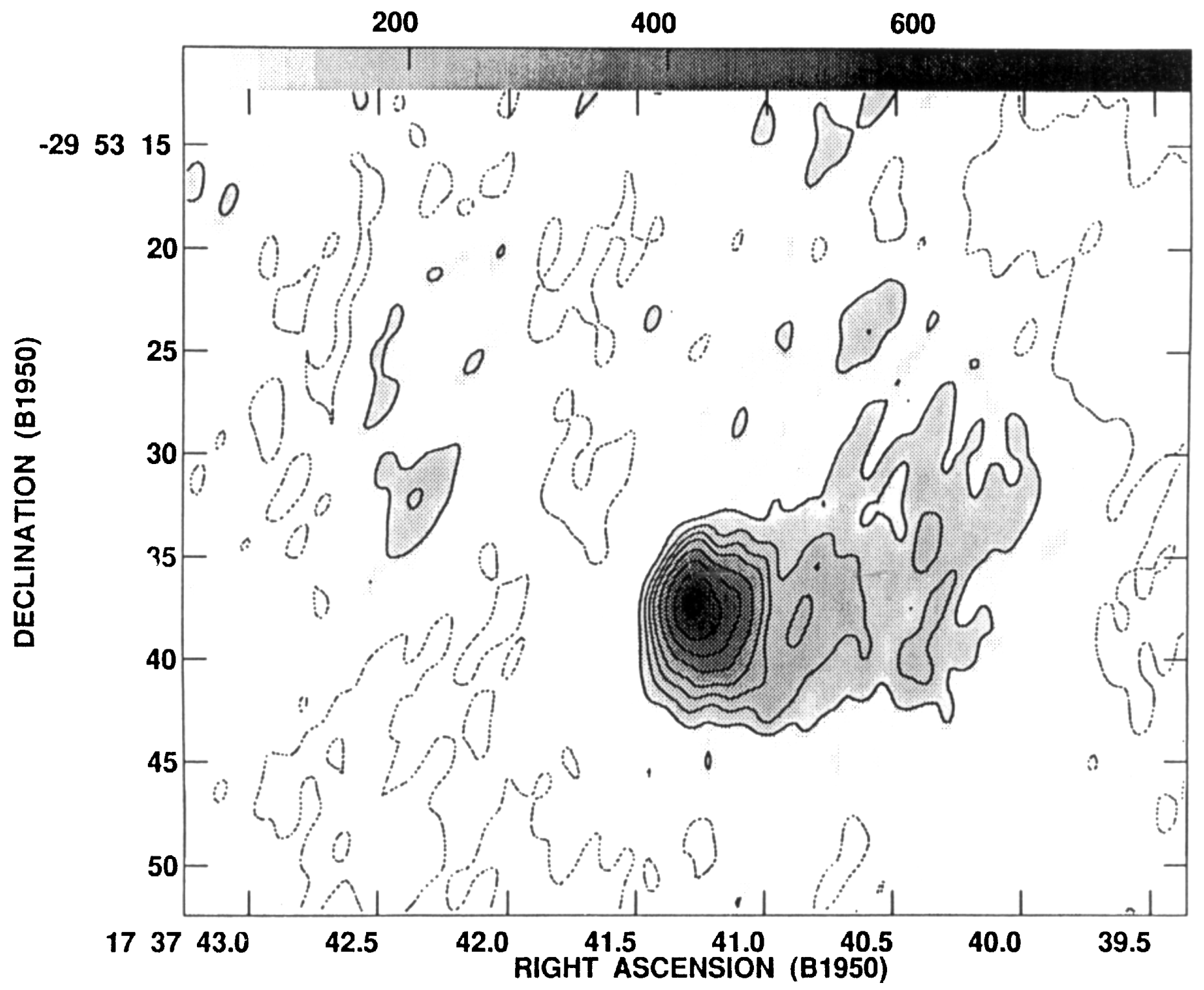

FIG. $4 a$

FIG. 4.-Magnified images at $20 \mathrm{~cm}(a)$ and $6 \mathrm{~cm}(b)$ of the region containing source 3 from the 1994 April observations. The $6 \mathrm{~cm}$ image has been convolved to the same resolution as that of the $20 \mathrm{~cm}$ image. The contours in the $20 \mathrm{~cm}$ image $(a)$ are at $-0.075,0.075,0.15,0.225,0.3,0.4,0.5$, and $0.6 \mathrm{mJy}$ beam $^{-1}$. The gray-scale flux density range is from 30 to $800 \mu \mathrm{Jy}$ beam ${ }^{-1}$. The contours in the $6 \mathrm{~cm}$ image $(b)$ are at $-0.1,0.1,0.15,0.2,0.25,0.3,0.35,0.4$, and $0.45 \mathrm{mJy}$ beam $^{-1}$. The gray-scale flux density range is from 50 to $500 \mu \mathrm{Jy} \mathrm{beam}^{-1}$.

of the VLA antennas are $30^{\prime}$ at $20 \mathrm{~cm}$ and $9^{\prime}$ at $6 \mathrm{~cm}$ in the A configuration. The largest angular scale structures "visible" to the array in the A configuration are $\approx 38^{\prime \prime}$ at $20 \mathrm{~cm}$ and $\approx 10^{\prime \prime}$ at $6 \mathrm{~cm}$. Data were taken in the spectral line mode with $50 \mathrm{MHz}$ bandwidth and three spectral channels. In both bands, data were acquired in dual polarization mode and at two frequencies: 1365 and $1435 \mathrm{MHz}$ in the $20 \mathrm{~cm}$ band and $4835 \mathrm{MHz}$ in the $6 \mathrm{~cm}$ band. Total integration time, including both days of observations, was approximately $4 \mathrm{hr}$ in each band. The flux density scale was set by using the primary calibrators $3 \mathrm{C} 48$ and $3 \mathrm{C} 286$, and the instrumental gain variations were corrected using interspersed observations of the secondary calibrator $1748-253$. The data were processed using the Astronomi- cal Image Processing System developed by the NRAO. Separate CLEANed images covering the entire field of view were constructed from observations on each day. In both the bands, four sources were detected in the vicinity of the gamma-ray source EXS 1737.9-2952. The images from the two days were then averaged since no significant difference was seen either in the structure or in the flux density of the four sources detected in the two images. The rms noise in the final images are $77 \mu \mathrm{Jy}$ beam $^{-1}$ at $20 \mathrm{~cm}$ and $23 \mu \mathrm{Jy}$ beam $^{-1}$ at $6 \mathrm{~cm}$. Some relevant parameters of the observations are given in Table 1.

The positions, flux densities, spectral indices, and angular sizes of the four detected sources (labeled 1 to 4 ) are given in Table 2. All the four sources are within the positional error 


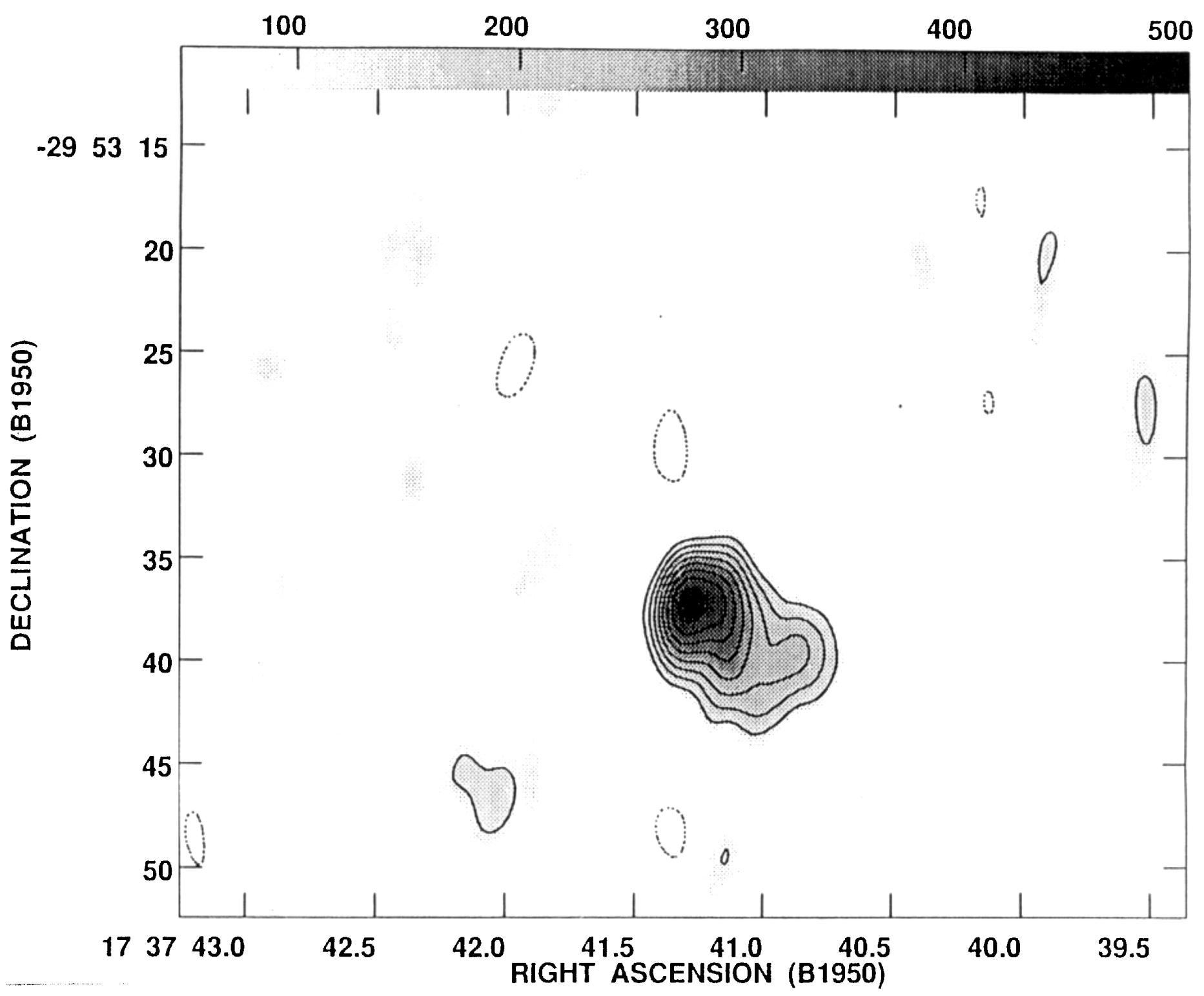

FIG. $4 b$

radius (10') of EXS 1737.9-2952. Figure 3 gives the location of the four sources and that of EXS 1737.9-2952. Sources 1,2 , and 4 are barely resolved in both 6 and $20 \mathrm{~cm}$ images, their angular sizes being $\approx 1^{\prime \prime}$. These three sources have a nonthermal, spectrum with a spectral index $\approx-0.85$ between 20 and $6 \mathrm{~cm}$. For these small diameter sources spectral index measurements using images with different resolutions are reasonably accurate. Source 3 is extended, has indications of having a head-tail morphology, and has a spectral index of -0.7 . Figures $4 a$ and $4 b$ give the magnified images of this source at $20 \mathrm{~cm}$ and $6 \mathrm{~cm}$, respectively. There is a marginal indication in the $20 \mathrm{~cm}$ image that source 3 may have a weak second component displaced about $15^{\prime \prime}$ northeast.

In analogy with 1E 1740.7-2942 (Mirabel et al. 1991) or GRS 1758-258 (Rodriguez, Mirabel, \& Mark 1992), we could look for a source with the morphology of a radio galaxy with a variable central source and radio lobes. On the basis of the observed morphology, flux density, or spectral index, it is not possible to firmly associate any of these four sources as a radio counterpart of the hard X-ray source EXS 1737.9-2952.

In order to investigate any possible variability in the four sources and thereby to look for a possible association with the X-ray source, we undertook further observations of this field during 1994 November and December. Observations were made at $6 \mathrm{~cm}$ in the VLA C configuration, which gave an angular resolution of $8.5 \times 5^{\prime \prime} .1$. The mode of observation was the same as in 1994 April; the 1994 December observations were 3 hours in length. The largest angular structure "visible" in this configuration was 120 ", which is more than a factor 10 larger compared to the 1994 April observations in the A configuration. The images made from the November and December data produced essentially identical results. All four sources detected in 1994 April were also detected in the November/December images, but 


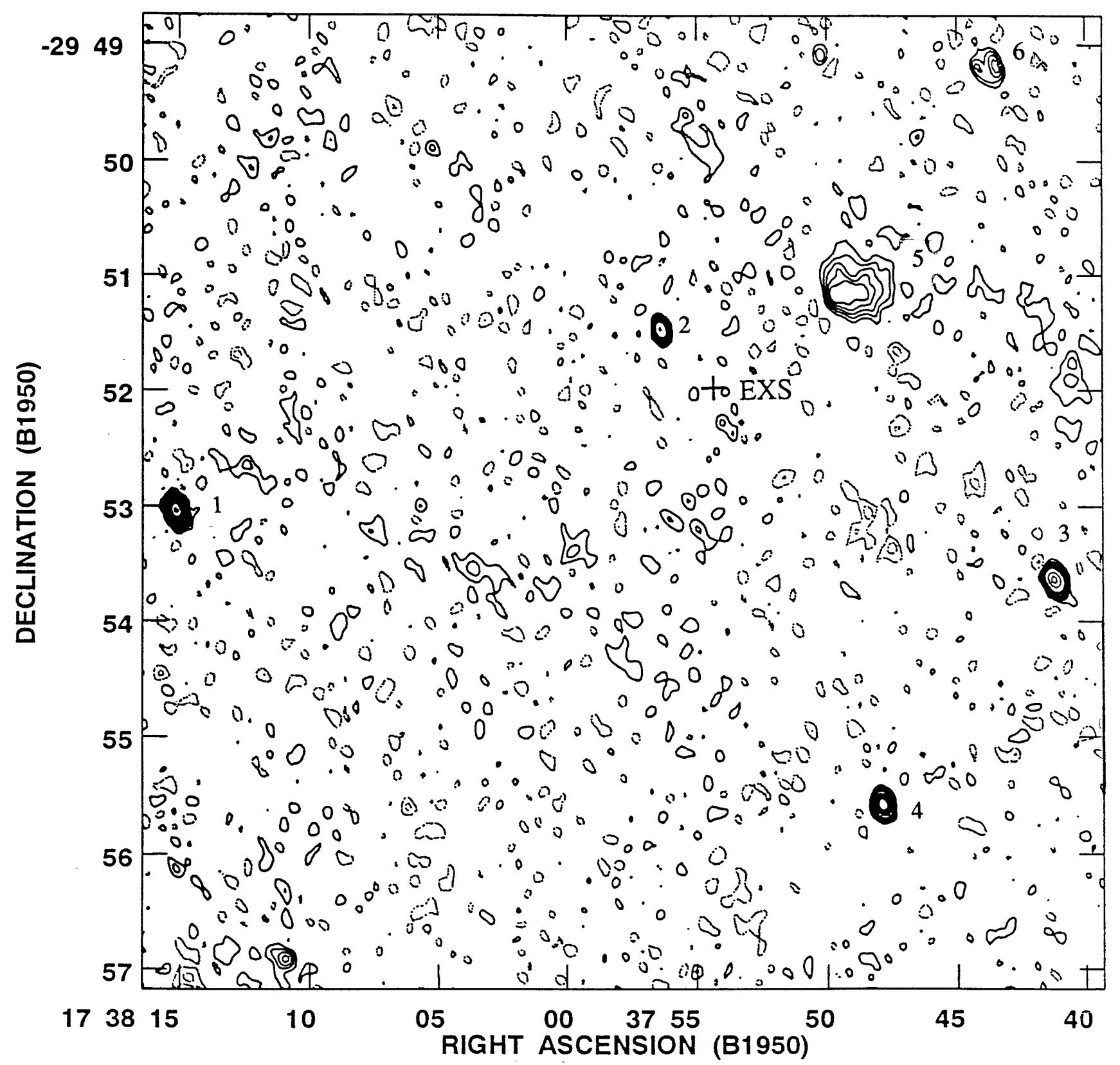

FIG. 5. $-6 \mathrm{~cm}$ image of the region containing EXS 1737.9-2952. This image was made by combining the observations made during 1994 November and December in the C configuration of the VLA. The contours are at $-0.08,-0.04,0.04,0.08,0.12,0.16,0.2,0.3,0.4,0.8,1.2,1.6,2,3,4$, and $6 \mathrm{mJy}^{-1}$ beam ${ }^{-1}$.

due to the coarser angular resolution, the sources were unresolved. There was no variation in the flux density of any of the four sources between 1994 November and December. A combined image from the two data sets is shown in Figure 5. In addition to the four sources seen in 1994 April, two other extended sources are seen in the northwest portion of Figure 5. Parameters of these two sources (labeled as 5 and 6) along with those of the other four are given in Table 2. Sources 5 and 6 were not detected at either $20 \mathrm{~cm}$ or $6 \mathrm{~cm}$ during 1994 April observations. Figure 6 shows a magnified image of this field obtained at $6 \mathrm{~cm}$ in 1994 November-December. The absence of detection of source 5 and 6 in 1994 April can be attributed to resolution and sensitivity effects. The A configuration observations of 1994 April at $6 \mathrm{~cm}$ are sensitive to struc- tures smaller than $10^{\prime \prime}$ and therefore sources 5 and 6 , which are $\approx 30^{\prime \prime}$ and $\approx 12^{\prime \prime}$, respectively, are not detected, although the rms noise in the image is comparable to that of Figure 6. On the other hand, the $20 \mathrm{~cm}$ image in Figure $4 b$ has more than 3 times the rms noise of the $6 \mathrm{~cm}$ image in Figure $4 a$ and furthermore it is sensitive to structures which are smaller than $38^{\prime \prime}$ compared to $120^{\prime \prime}$ observable in Figure $4 a$. For these reasons the absence of sources 5 and 6 in 1994 April is not surprising.

\section{MILLIMETER OBSERVATIONS}

We used the low-resolution CO (1-0) survey of extended regions, including EXS, shown in Figure 7, performed by $\mathbf{J}$. Bally (1993, private communication), to set up our observing program. This map shows that the EXS error box is 


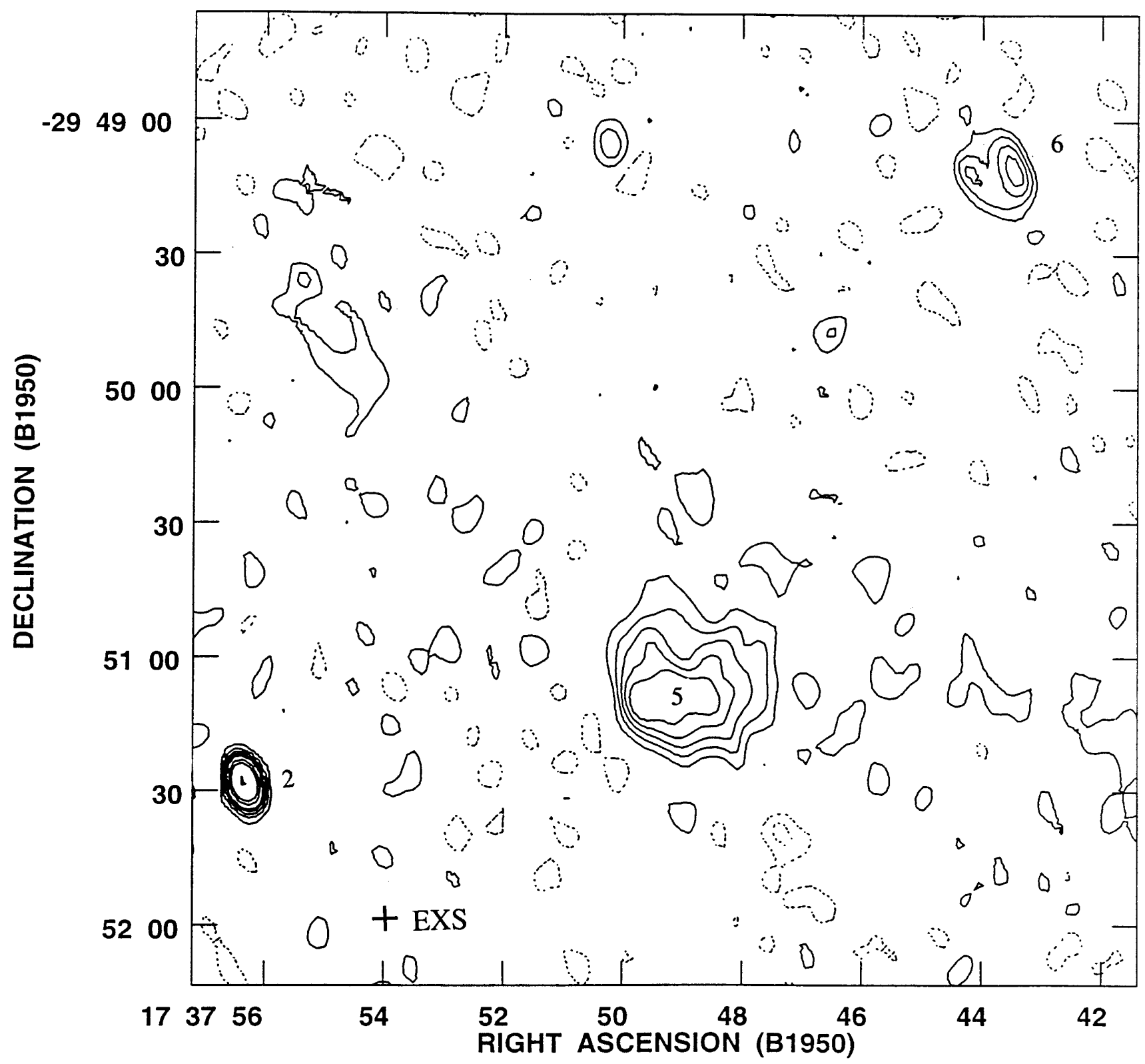

FIG. $6 a$

FIG. 6.-Magnified images of the region containing sources 5 and 6 obtained at $6 \mathrm{~cm}$ in 1994 November-December. The contours are at $-0.08,-0.04$, $0.04,0.08,0.12,0.16,0.2,0.3,0.4$, and $0.8 \mathrm{mJy}_{\text {beam }}{ }^{-1}$.

spatially coincident with a molecular cloud. In order to study this cloud in more detail, we conducted millimeter observations in 1993 August using the Swedish-ESO submillimeter telescope (SEST; La Silla, Chile). ${ }^{12} \mathrm{CO}(1-0)$ and ${ }^{13} \mathrm{CO}(1-0)$ transition lines at 115 and $110 \mathrm{GHz}$, respectively, were used for mapping and estimating the column density (assuming LTE). The $3 \mathrm{~mm}$ receiver system consisted of two cooled Schottky mixers and covered the frequency band $80-120 \mathrm{GHz}$. The spectra were analyzed simultaneously by two high $(0.004 \mathrm{MHz})$ and low $(0.7 \mathrm{MHz})$ resolution acoustic-optic spectrometers. The half-power beam size (FWHM) was $44^{\prime \prime}$ at $115 \mathrm{GHz}$. The background was eliminated by position switching. Due to the $10^{\prime}$ uncertainty in the EXS location, we carried out a survey first in
${ }^{12} \mathrm{CO}$, scanning a grid of $45^{\prime \prime}$ pixels $27 \times 16$ with 30 s integration time centered on the EXS error box. We therefore obtained a ${ }^{12} \mathrm{CO}$ map of the region and then surveyed the region centered on the maximum of the previous ${ }^{12} \mathrm{CO}$ emission, in ${ }^{13} \mathrm{CO}(10 \times 10$ pixels $), 60 \mathrm{~s}$ integration time. The spectra are shown in Figures 8 and 9 (preliminary results were published in Durouchoux et al. 1993 and Vilhu et al. 1994).

We found a cloud at the "forbidden" velocity of +135 $\mathrm{km} \mathrm{s}^{-1}$ (moving in the opposite direction to the central bar in the Galactic center region), close to the EXS source $\left(l^{\mathrm{II}}=-1^{\circ} 34, b^{\mathrm{II}}=+0^{\circ} 34\right)$ in both ${ }^{12} \mathrm{CO}(1-0)($ Fig. 10) and ${ }^{13} \mathrm{CO}(1-0)$ (Fig. 11). The width of the lines in ${ }^{12} \mathrm{CO}$ and ${ }^{13} \mathrm{CO}$ is about $15 \mathrm{~km} \mathrm{~s}^{-1}$, compatible with a massive molec- 


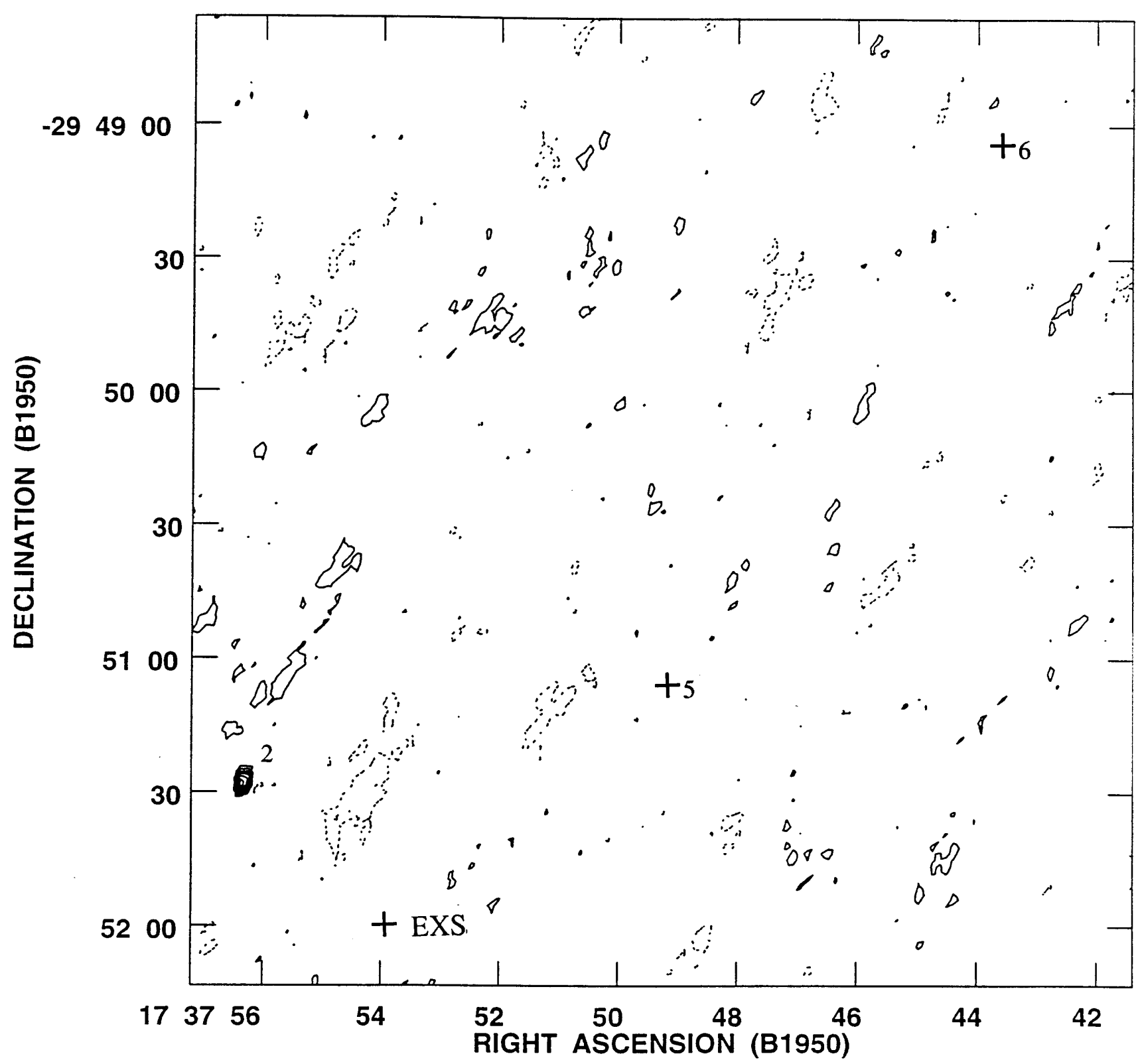

FIG. $6 b$

ular cloud. Two cloud velocity components may be seen: a complex in the $-20 \mathrm{~km} \mathrm{~s}^{-1}$ to $+60 \mathrm{~km} \mathrm{~s}^{-1}$ range attributed to the GC clouds and a velocity component centered at $V_{\mathrm{LSR}}=135 \mathrm{~km} \mathrm{~s}^{-1}$. Estimates of the maximum column density and cloud mass are $8 \times 10^{21} \mathrm{~cm}^{-2}$ (Fig. 12) and $1.5 \times 10^{4} M_{\odot}$, respectively, from the ${ }^{13} \mathrm{CO}(1-0) /{ }^{12} \mathrm{CO}$ (1-0) ratio, assuming LTE (following the method by Gahm, Johansson \& Liseau 1993). The cloud density in this region has been estimated at $7 \times 10^{2} \mathrm{~cm}^{-3}$ (for a $3 \mathrm{pc}$ size). Since most molecular clouds have filling factors of about $10 \%$, the density at condensation locations, if present, might be of the order of $7 \times 10^{3} \mathrm{~cm}^{-3}$.

Further investigations were conducted in 1994 at Daewa Korean Observatory (South Korea) using the $\mathrm{HCO}+(1-0)$ $89.19 \mathrm{GHz}$ transition and at Mauna Kea with the JCMT using the CS (5-4) transition $(224.94 \mathrm{GHz})$ in order to search for higher density condensations. Both results were negative, proving that the cloud density does not exceed $10^{4}$ $\mathrm{cm}^{-3}$ and that the ionization state is low, which can be explained by a low state or even a complete extinction of the possible X-ray counterpart.

\section{EINSTEIN AND IRAS MAPS}

We searched in the archived Einstein maps, within the $5 \sigma$ EXS error circle, for soft X-ray sources which could be the counterpart of EXS. We found in the Einstein IPC field I 2517 (Fig. 13) a 0.084 counts $\mathrm{s}^{-1}$ "source" $(4 \sigma)$, which is probably due to a statistical effect, but would, however, aid attention to harder X-ray energies. A nearby pulsar (PSR 1737-30) is outside the EXS error box (Fig. 13).

An optical image of the EXS region is presented in Figure 14. Two stars are marked for reference: one of the many 


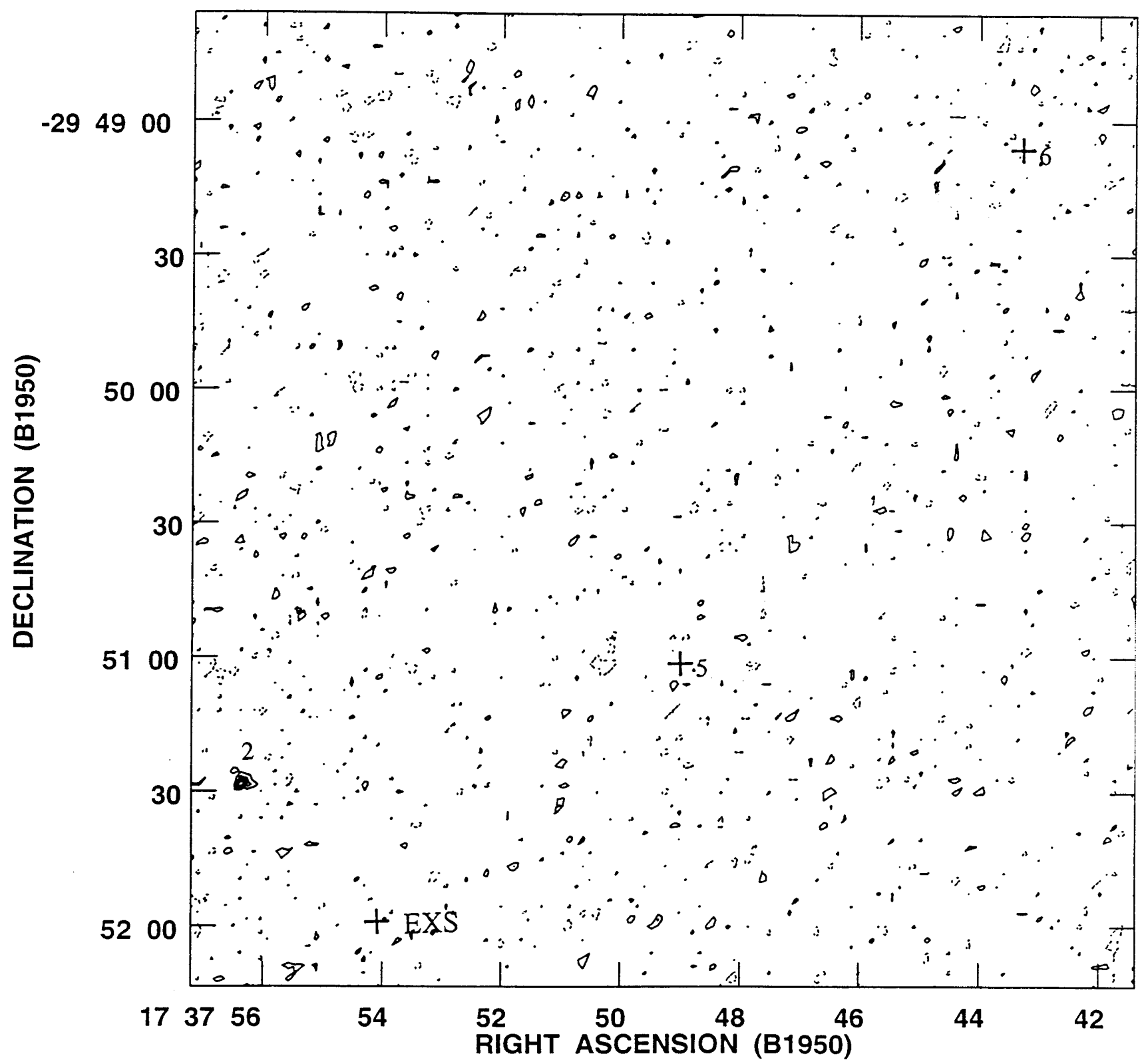

FIG. $6 c$

Hubble guide stars (GSC 06839-0055, magnitude 11.6) and SAO $165622(V=7.6)$, but both are outside the EXS error box.

We also searched for possible IR emission in the EXS region by using high-resolution IR maps from the NASA Infrared Astronomical Satellite (IRAS).

Nothing in the four maps at $12,25,60$ and $100 \mu \mathrm{m}$ is visible (see Fig. 15 for the $60 \mu \mathrm{m}$ maps) at the location of the six radio candidates (labeled 1-6 in the Table 2). The large complex located on the southwest and the IRAS $17375-30000$ source are both outside the source position error circle.

\section{DISCUSSION AND CONCLUSION}

From our VLA observations, a comparison of the measured flux densities of sources 1-4 (Table 2) shows that there has been no variation between 1994 April, 1994 November, and 1994 December. There is also no evidence for any change in the structure of these sources. From the general properties of these sources, they appear to belong to the category of extragalactic radio sources. On the basis of these observations, it is not possible for us to make any clear association between the X-ray source and the radio sources. Further observations of source 3 (in A configuration) and those of sources 5 and 6 in the $C$ or D configurations may reveal additional aspects such as variability or change in structure which help in making a possible association.

It is not well established if a compact source (not belonging to a binary system) is able to accrete matter from a dense cloud and produce X-ray photons in an accretion disk. The clumpiness of the cloud could explain X-ray flares seen in sources where a stellar companion has not been detected (e.g., 1E and GRS 1758 sources). Of course, AGNs 


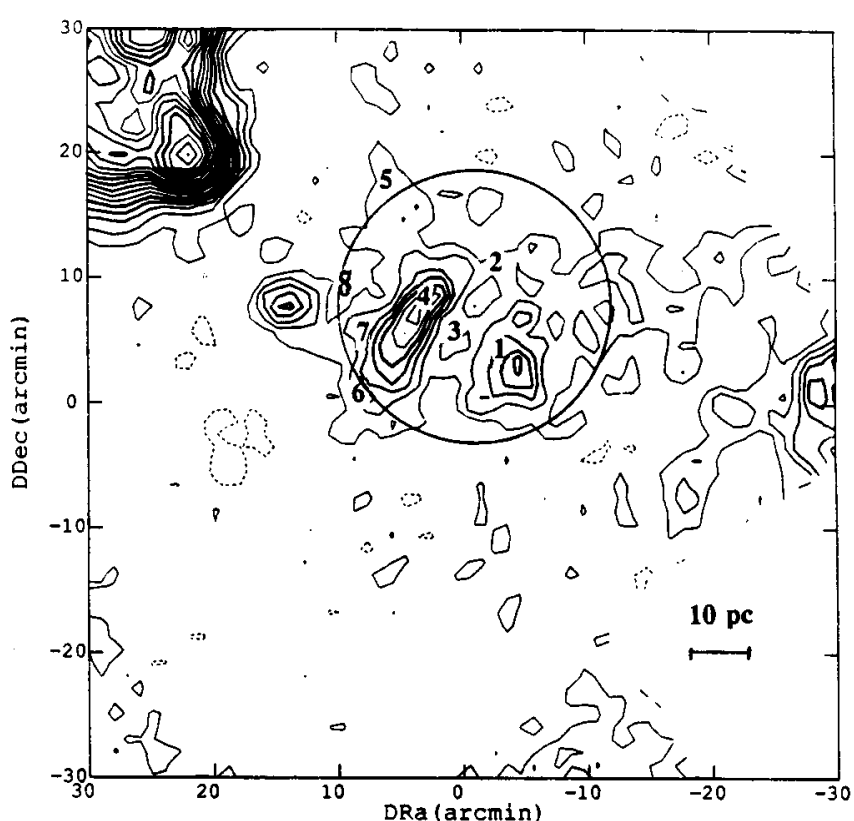

FIG. 7.- ${ }^{12} \mathrm{CO}(1-0)$ survey (in the velocity range $130-140 \mathrm{~km} \mathrm{~s}^{-1}$ ) of EXS region from J. Bally (1993, private communication). The circle represents the $5 \sigma$ EXS error box. The superimposed radio sources (quoted 1 to 8) are from a $20 \mathrm{~cm}$ VLA survey (Gray et al. 1993). The source fluxes, in $\mathrm{mJy}$, are (1) 6.6 ; (2) 2.0 , variable; (3) 1.3 ; (4) 24.3 , flat spectrum, compact $\mathrm{H}$ II region; (5) 1.9 ; (6) 6.9 ; (7) 1.2 ; (8) 4.4 .

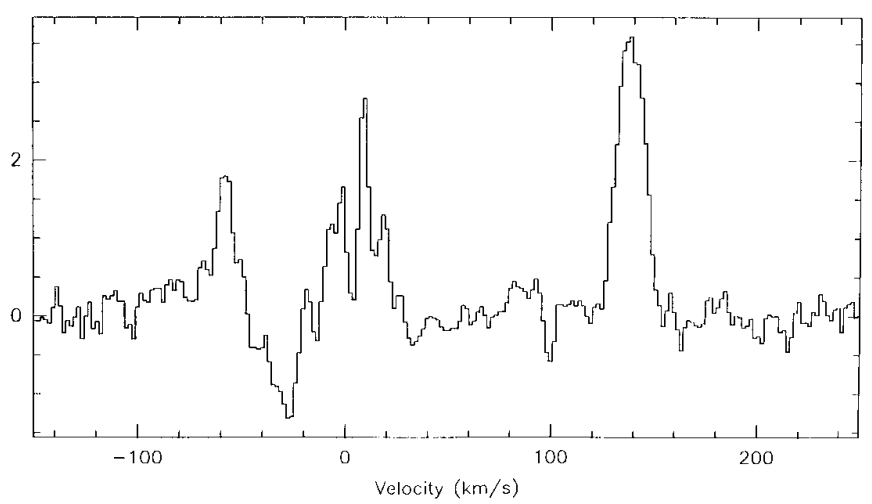

FIG. 8. $-{ }^{12} \mathrm{CO}(1-0)$ spectrum in the direction of EXS 1737.9-2952

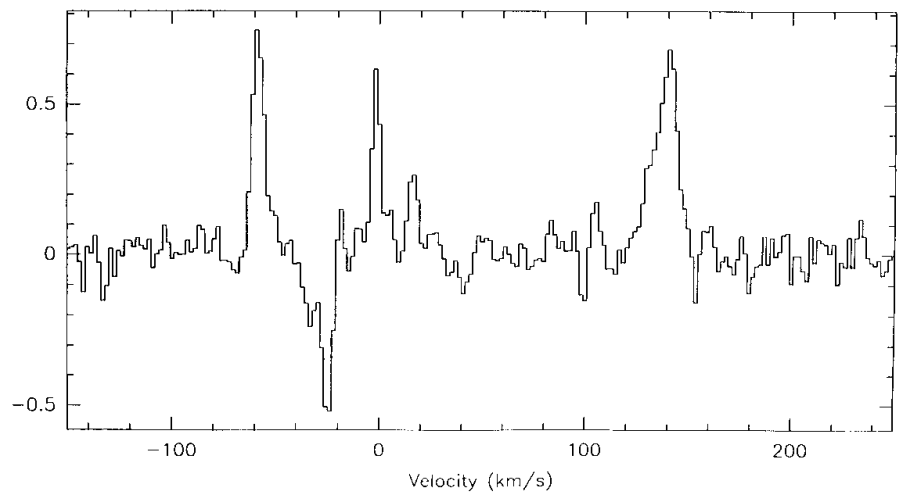

Fig. 9. $-{ }^{13} \mathrm{CO}(1-0)$ spectrum in the direction of EXS 1737.9-2952

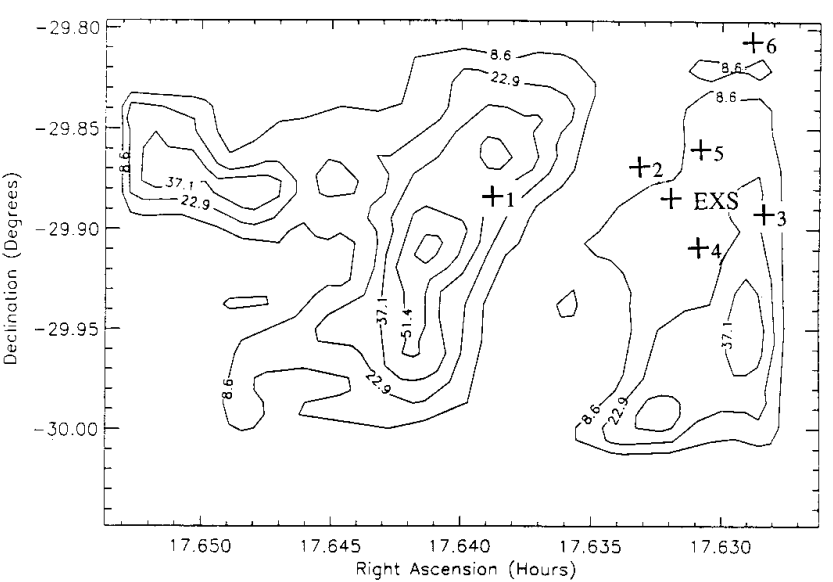

FIG. 10.-Contour map at ${ }^{12} \mathrm{CO}(1-0) 115.2712 \mathrm{GHz}$, in the vicinity of EXS $1737.9-2952$, in the velocity range $120-170 \mathrm{~km} \mathrm{~s}^{-1}$. The location of the six radio sources as referenced in Table 2 are indicated with a plus sign.

are compact objects accreting matter from their surrounding media, but the mass of the central nucleus is many orders of magnitude larger than the stellar black hole possibly associated with EXS.

One must underline that the Galactic sources with a behavior characterized by different states mostly belong to the Galactic center or Galactic disk regions (excluding $\mathrm{X}$-ray novae), where the detection of a companion is very difficult due to the high absorption. On the other hand, the sources out of the Galactic plane, where those different states have been detected, are commonly binary systems.

We searched for dense media associated with well known binaries such as Cyg X1, Nova Muscae, and a few others (in $\mathrm{CO}$ and ${ }^{13} \mathrm{CO}$ lines), but the lack of such detection could indicate either that all the flaring sources are part of a binary system, sometimes with a companion unobserved due to absorption reasons, or that a single compact object associated with a dense medium is able to accrete matter.

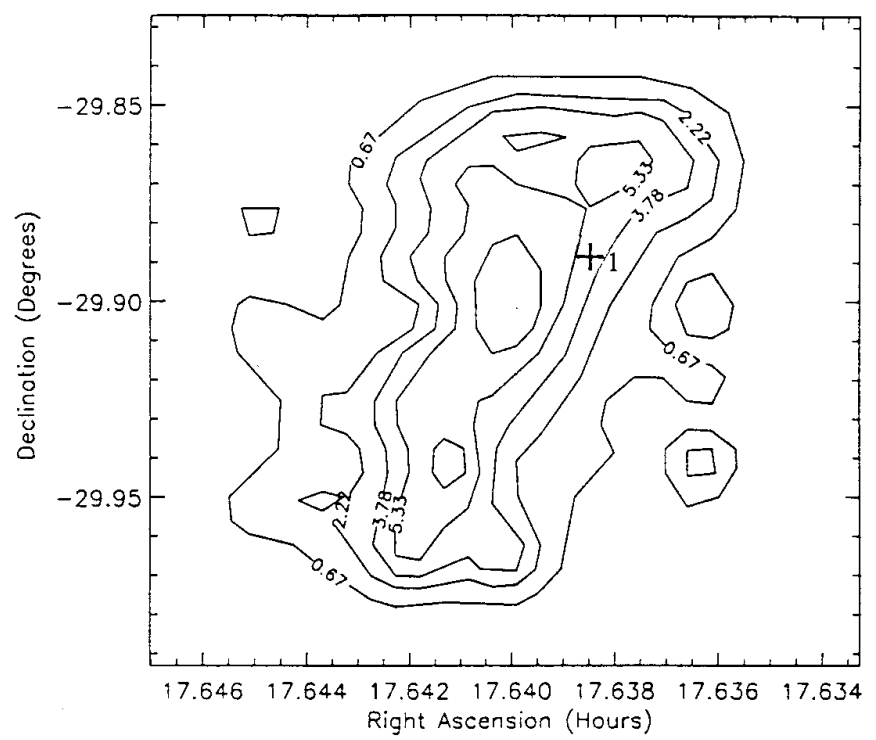

FIG. 11.-Contour map at ${ }^{13} \mathrm{CO}(1-0) 110 \mathrm{GHz}$, in the vicinity of EXS $1737.9-2952$, in the velocity range $120-170 \mathrm{~km} \mathrm{~s}^{-1}$. The location of the VLA candidates are indicated. 


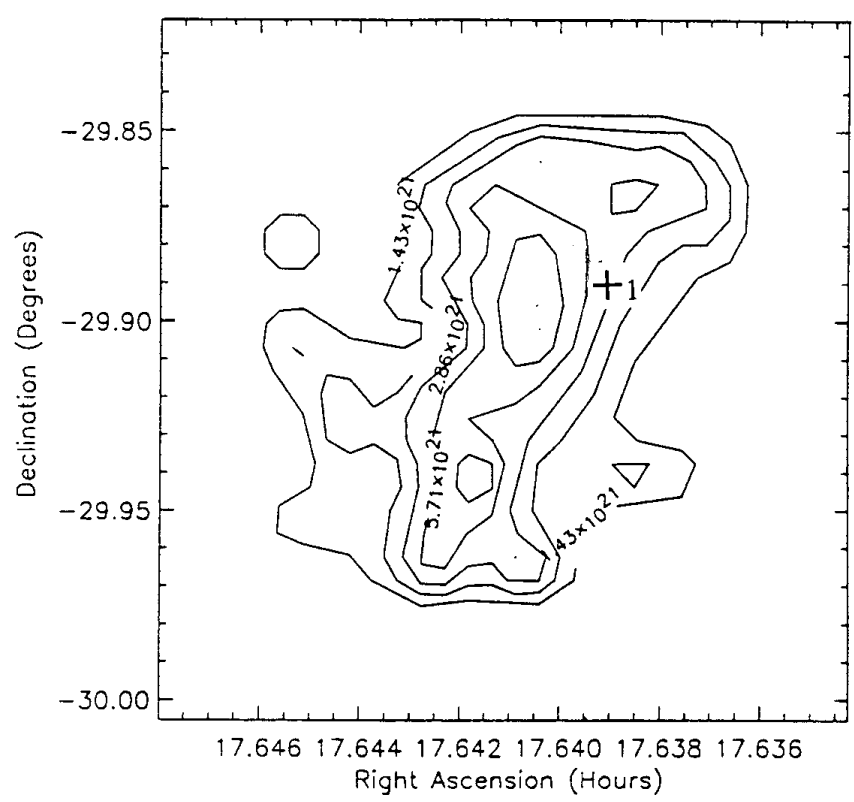

FIG. 12.-EXS 1737.9-2952, $\mathrm{H}_{2}$ column density. The contours are labeled by the column density (maximum $=10^{22} \mathrm{~cm}^{-2}$ ). Assuming a distance of $8.5 \mathrm{kpc}$, the mean density is $\approx 10^{3} \mathrm{~cm}^{-3}$.

Is EXS 1737.9-2952 a new, hard X-ray transient associated with a molecular cloud, which could provide the matter to feed the black hole as well as the annihilation site for a narrow annihilation line? Our observations could favor such a scenario; so do the similarities with $1 \mathrm{E}$, even though no jets have ever been detected in the case of EXS. Mineshige, Soon-Wook \& Wheeler (1990) pointed out that in the case of a disk instability without sources of heat external to the disk, the disk becomes thermally unstable for a mass transfer rate of the order of $M=10^{17.0} \mathrm{~g} \mathrm{~s}^{-1}\left(10^{-8.8}\right.$ $M_{\odot} \mathrm{yr}^{-1}$ ). This predicted instability might exhibit relaxation-type behavior with quiescent periods on the order of $1 \mathrm{yr}$, which could be what we observed by chance in EXS.

This estimated accretion rate corresponds to a luminosity, $L_{\mathrm{X}}=7 \times 10^{38} \mathrm{ergs} \mathrm{s}^{-1}$, and the measured 20-200 $\mathrm{keV}$ luminosity (assuming EXS to be at the GC distance, 8.5 $\mathrm{kpc}$ ) is $7 \times 10^{37} \mathrm{ergs} \mathrm{s}^{-1}$, taking a $10 \% \epsilon$ efficiency. The $35-75 \mathrm{keV}$ SIGMA sensitivity is about $5 \mathrm{mCrab}(3 \sigma)$, which corresponds to a luminosity at the GC distance of $2.6 \times 10^{35} \mathrm{ergs} \mathrm{s}^{-1}$. The lack of detection of any X-ray source at the EXS position in the SIGMA plates can have different explanations: (1) the occurrence of these X-ray outbursts compared with the frequency of the Galactic center region observations performed by SIGMA (approximately 1 or 2 times a year for observations lasting about 1 month), and (2) the moderate sensitivity of the SIGMA instrument (if spherical accretion is considered, it might be less powerful than standard Bondi-Hoyle accretion, and consequently one needs more sensitive instruments to detect it).

Concerning the other gamma-ray satellites (HEAO 3, $C G R O)$, the poor angular resolution of the instruments

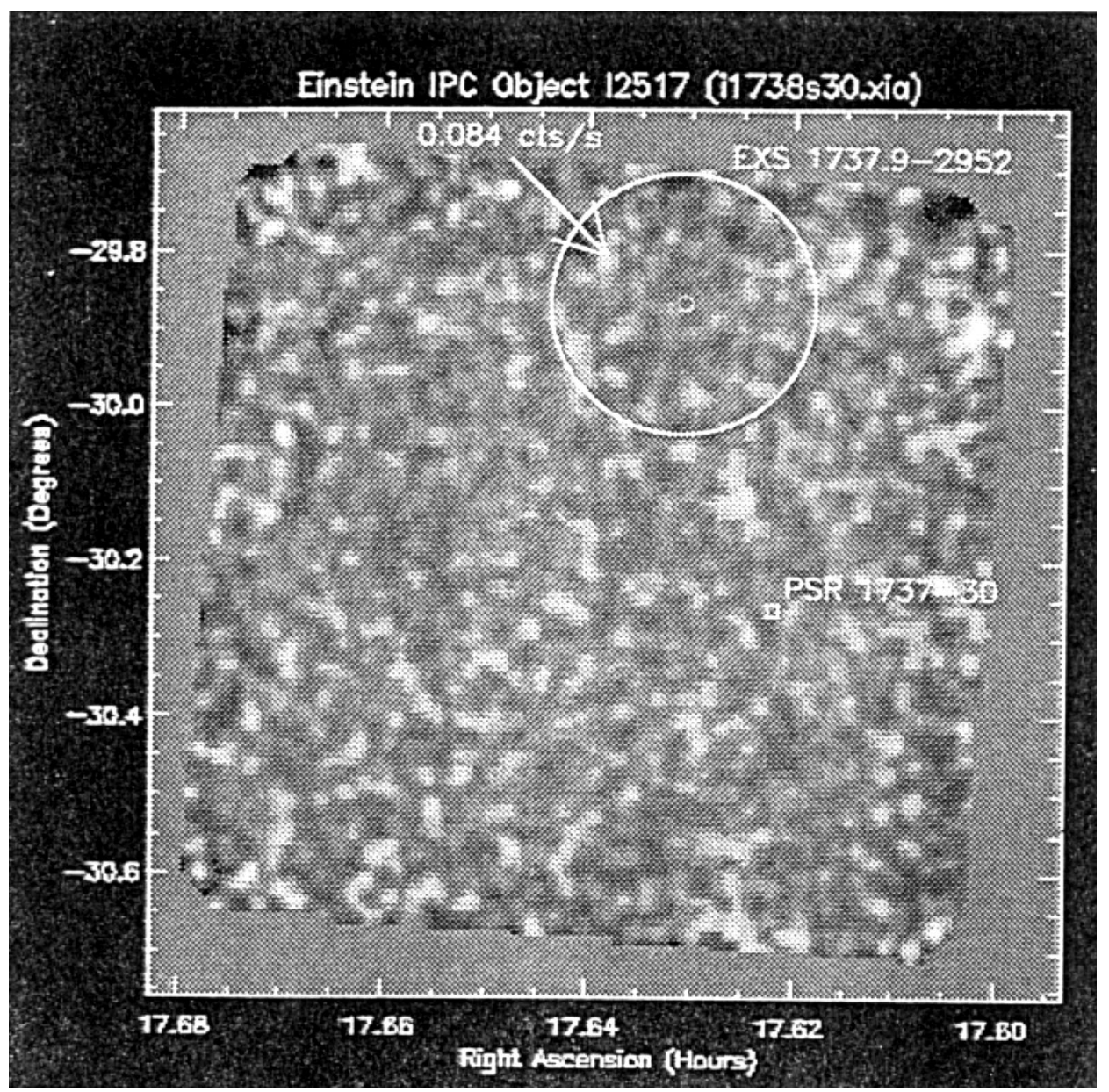

FIG. 13.-Einstein IPC field (I 2517) with EXS error circle ( $t_{\exp }=30$ minutes). The "source" marked with an arrow $\left(0.0084\right.$ count s $\left.{ }^{-1}\right)$ seems to be a marginal enhancement. Also visible on the figure is the PSR 1737-30 location. 


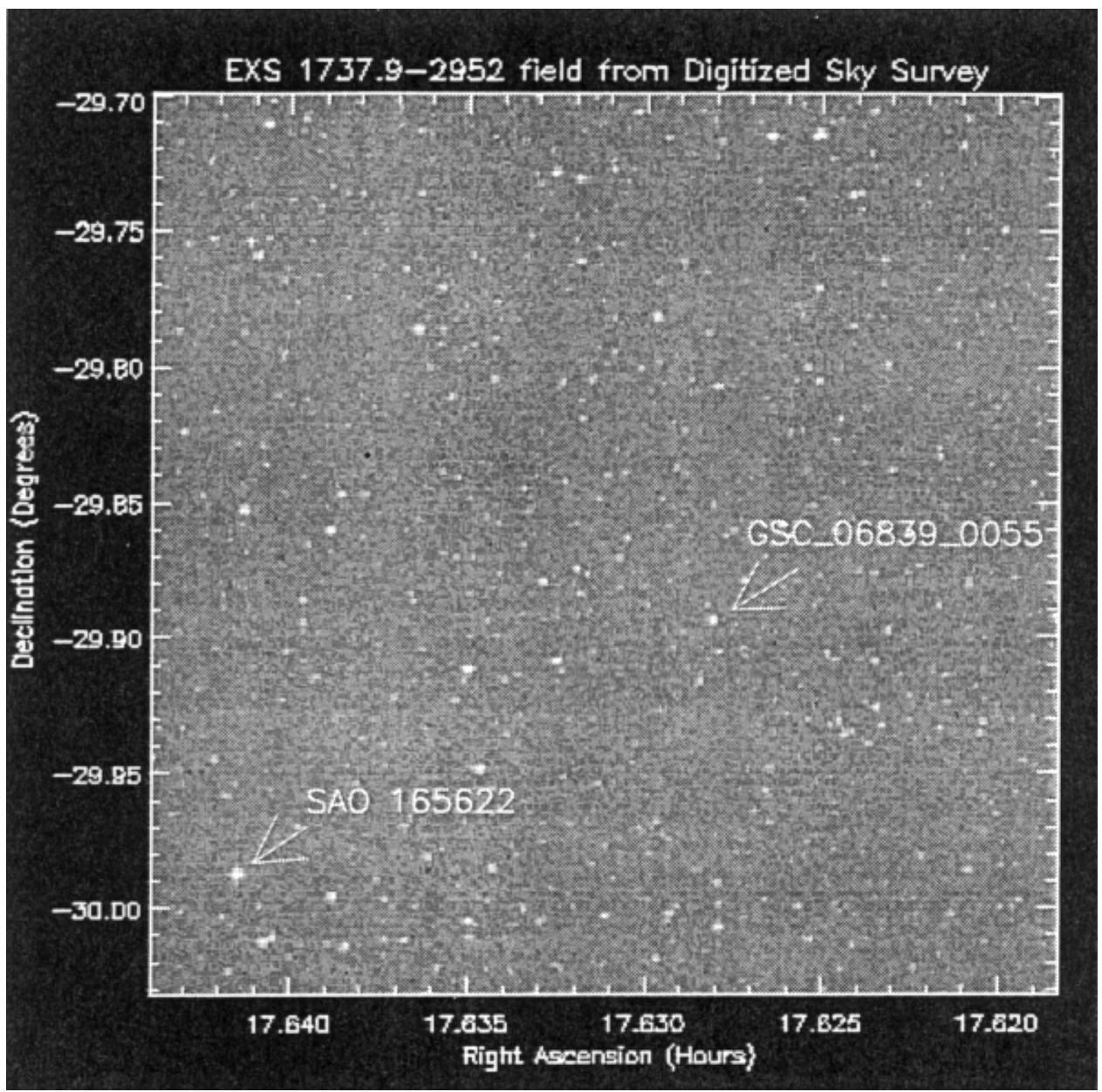

FIG. 14.- Optical image of the EXS region. Two stars are indicated: GSC 06839-0055 from the Hubble guide stars and SAO 165622.

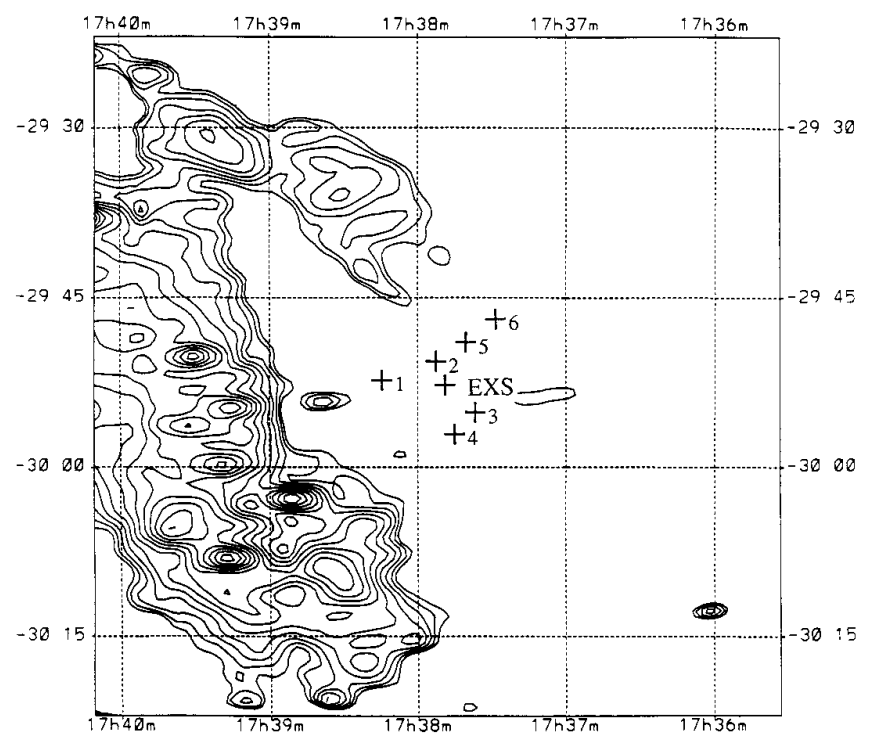

FIG. 15.- - High-resolution IRAS map of the EXS 1737.9-2952 region: $\lambda=60 \mu \mathrm{m}$. Contour intervals are multiples of 1.4140 from 105.59 to 6745.5 $\mathrm{mJy} \mathrm{sr}^{-1}$. The location of the six radio sources as referenced in Table 2 are indicated with plus signs. onboard these satellites could have made an EXS outburst attributed to $1 \mathrm{E}$ source which is only located $40^{\prime}$ away.

Finally, Campana \& Pardi (1993) have estimated the present number density of black holes as $8.0 \times 10^{-4} \mathrm{pc}^{-3}$, taking Salpeter's (1955) initial mass function (IMF), an integrated Galactic black hole birth rate of $8.5 \times 10^{-3} \mathrm{yr}^{-1}$, and $10 M_{\odot}$ as a lower limit to the mass of black hole progenitors $\left(M_{\mathrm{BH} \text { prog }}\right)$. Taking a more realistic value for $M_{\mathrm{BH} \text { prog }}\left(25 M_{\odot}\right)$ and about $0.5-7$ such objects per molecular cloud, X-ray and gamma-ray measurements performed with a sensitive instrument having high-energy resolution and imaging capabilities (INTEGRAL) would help to decide whether or not black holes residing in dense clouds are able to explain what has been seen in 1E, GRS $1758-258$, and possibly EXS sources. If the frequency of occurrence of this high state is related to the number of high-density clumps in the cloud, which might be low, this is also an argument to explain the lack of detection by SIGMA during their fall 90 GC survey. Nevertheless, GRIS (the GSFC high-energy resolution balloon spectrometer) observed the same region 8 days after the EXS detection and did not detect any feature (GRIS integrates the 
$\mathrm{X}$ /gamma-ray flux over a relatively large FOV). It seems difficult but not totally impossible to have a temperature increase followed by a decrease in such a short period of time. The idea of positron annihilation in an accretion disk was first proposed by Ramaty et al. (1992), and the high density and relatively low temperature of the inner region of an accretion disk makes it an ideal, or at least a possible, place for the high-energy positrons intercepted by the disk to slow down and annihilate within a few $r_{s}$ (Chen \& Gehrels 1993). Therefore molecular clouds are no longer needed to explain the observed features.
We appreciate the open policy of the National Radio Astronomy Observatory, which is operated by Associated Universities, Inc., under cooperative agreement with the US National Science Foundation. The authors are also much indebted to the SEST staff, in particular to Lewis Knee and Francisco Azagra for their competent support during the observations as well as the Daeduk Radio Observatory for conducting radio observations of EXS region. We finally wish to acknowledge Ian Smith for helpful comments on the manuscript.
Bally, J., \& Leventhal, M. 1991, Nature, 353, 324

Campana, S., \& Pardi, C. 1993, A\&A, 277, 477

Chen, W., Gehrels, N., \& Leventhal, M. 1993, ApJ, 403, L71

Durouchoux, P., Vilhu, O., Wallyn, P., Huovelin, J., \& Bally, J. 1993, in AIP Conf. Proc. 304, ed. C. Fichtel, N. Gehrels, \& J. Norris (New York: AIP), 436

Gahm, G. F., Johansson, L. E. B., \& Liseau, R. 1993, A\&A, 274, 415

Gray, A. D., Whitehook, J. B. Z., Cram, L. E., \& Goss, W. M. 1993, ApJ, 264,678

Grindlay, J. E., Covault, C. E., \& Manandhar, R. P. 1993, A\&AS, 97, 155

Liang, E. P. 1991, ApJ, 367, 470

Lingenfelter, R., \& Hua, X. 1991, ApJ, 381, 426

Mahoney, W. A., et al. 1993, ApJ, 92, 387

Matteson, J., et al. 1993, A\&AS, 97, 185

Mirabel, I. F., Morris, M., Wink, J., Paul, J., \& Cordier, B. 1991, A\&A, 251, L43

Mineshige, S., Soon-Wook, K., \& Wheeler, J. C. 1990, ApJ, 358, L5

\section{REFERENCES}

Purcell, W. R., et al. 1996, Meeting of the AAS High Energy Astrophysics Division, 29 April-4 May (San Diego, CA)

Ramaty, R., Leventhal, M., Chan, K. W., \& Lingenfelter, R. E. 1992, ApJ, 392, L63

Rodriguez, L. F., Mirabel, I. F., \& Mark, J. 1992, ApJ, 401, L15

Salpeter, E. E. 1955 , ApJ, 121, 161

Shakura, N. I., \& Sunyaev, R. A. 1973, A\&A, 24, 337

Vilhu, O., Durouchoux, P., Wallyn, P., Huovelin, J., \& Bally, J. 1994, in New Horizon of X-Ray Astronomy: First Results from ASCA, ed. F. Makino \& T. Ohashi (Tokyo: Universal Academic Press), 445

Wallyn, P., \& Durouchoux, P. 1994, ApJ, 414, 178

Wallyn, P., Ling, J. C., Mahoney, M. A., Wheaton, W. A., Durouchoux, P. Astier-Perret, L., \& Poirot, L. 1996, in Proc. 2nd INTEGRAL Workshop, ed. C. Winkler, T. Courvoisier, \& P. Durouchoux (ESA SP 382; Noordwijk: ESA), 109

Zdziarski, A., et al. 1994, ApJ, 436, 762 